\title{
ON APPROACH To DECREASE DIMENSIONS OF FIELD-EFFECT TRANSISTORS FRAMEWORK ELE- MENT OF SRAM WITH INCREASING THEIR DIMENSIONS
}

\author{
E.L. Pankratov, E.A. Bulaeva \\ Nizhny Novgorod State University, 23 Gagarin avenue, Nizhny Novgorod, 603950, \\ Russia
}

\begin{abstract}
In this paper we consider manufacturing of elements SRAM with increased density of field-effect transistors consisting these elements. The approach based on manufacturing of the elements in heterostructure with specific configuration. We consider doping of several required areas of the heterostructure by diffusion or by ion implantation. After that dopant and radiation defects have been annealed framework optimized scheme.
\end{abstract}

\section{KEYWORDS}

Field-effect transistor, decreasing of dimension of transistors, element SRAM, increasing if density of elements of SRAM

\section{INTRODUCTION}

In the present time several actual problems of the solid state electronics (such as increasing of performance, reliability and density of elements of integrated circuits) are intensively solving. At the same time with increasing of density of elements of integrated circuits their dimensions should be decreased. Dimensions of these elements could be decreased by manufacturing in thin films heterostructures [1-4]. In this case it could be used inhomogeneity (layering) of heterostructures. To decrease dimensions of elements of integrated circuits are could be also used laser or microwave types of annealing [5-7]. These types of annealing give a possibility to obtain inhomogenous distribution of temperature in the considered samples or heterostructures during doping due to temperature. In this situation one can obtain decreasing dimensions of elements of integrated circuits. It should be noted, that radiation processing of doped materials also leads to changing their properties $[8,9]$.

In this paper we consider manufacturing element SRAM [10]. To manufacture the element we consider a heterostructure, which includes into itself a substrate and an epitaxial layer. Several sections are presented in the epitaxial layer. These sections were manufactured by using another materials. These sections have been doped. The doping has been done to produce required types of conductivity ( $p$ or $n$ ) in the considered sections. These sections have been doped by diffusion or by ion implantation. These areas became sources, drains and gates of transistors framework element SRAM (see Fig. 1). We consider annealing of dopant and/or radiation defects after the doping. Main aim of the present paper is analysis of changing of concentrations of dopants and radiation defects in space and time to determine conditions, which correspond to decreasing of dimensions of transistors framework element SRAM and at the same time to increase of these transistors.

DOI:10.5121/ijfcst.2016.6402 
International Journal in Foundations of Computer Science \& Technology (IJFCST) Vol.6, No.4, July 2016

\section{Method OF SOLUTION}

We determine distribution of concentration of dopant in space and time as the solution of the following equation

$$
\frac{\partial C(x, y, z, t)}{\partial t}=\frac{\partial}{\partial x}\left[D \frac{\partial C(x, y, z, t)}{\partial x}\right]+\frac{\partial}{\partial y}\left[D \frac{\partial C(x, y, z, t)}{\partial y}\right]+\frac{\partial}{\partial z}\left[D \frac{\partial C(x, y, z, t)}{\partial z}\right],
$$

which correspond to the following boundary and initial conditions

$$
\left.\frac{\partial C(x, y, z, t)}{\partial x}\right|_{x=0}=0,\left.\frac{\partial C(x, y, z, t)}{\partial x}\right|_{x=L_{x}}=0,\left.\frac{\partial C(x, y, z, t)}{\partial y}\right|_{y=0}=0,\left.\frac{\partial C(x, y, z, t)}{\partial y}\right|_{x=L_{y}}=0,
$$

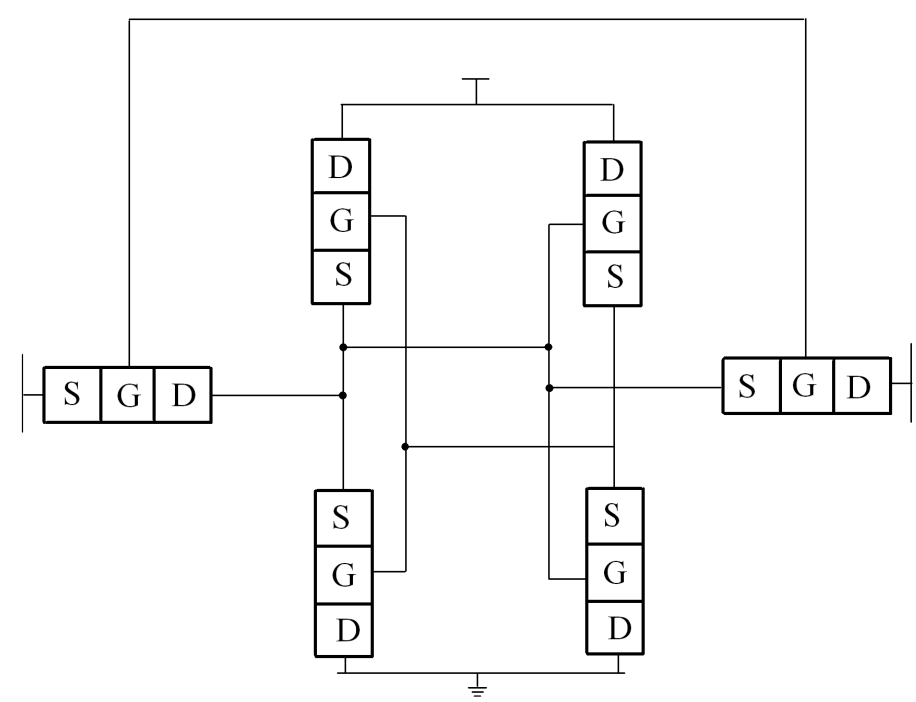

Fig. 1. Structure of element of SRAM. View from top

$$
\left.\left.\frac{\partial C(x, y, z, t)}{\partial z}\right|_{z=0}=0,\left.\frac{\partial C(x, y, z, t)}{\partial z}\right|_{x=L_{z}}=0, C x, y, z, 0\right)=f_{C}(x, y, z) .
$$

The function $C(x, y, z, t)$ describes distribution of concentration of dopant in space and time; $T$ is the temperature of annealing; $D_{C}$ is the dopant diffusion coefficient. Diffusion coefficient will be changed with changing of materials of heterostructure. Diffusion coefficient will be also changed with changing of temperature of annealing (with account Arrhenius law). Concentrational dependences of dopant diffusion coefficient could be approximated by the following relation [1012]

$$
D_{C}=D_{L}(x, y, z, T)\left[1+\xi \frac{C^{\gamma}(x, y, z, t)}{P^{\gamma}(x, y, z, T)}\right]\left[1+\varsigma_{1} \frac{V(x, y, z, t)}{V^{*}}+\varsigma_{2} \frac{V^{2}(x, y, z, t)}{\left(V^{*}\right)^{2}}\right] .
$$

The function $D_{L}(x, y, z, T)$ describes dependences of dopant diffusion coefficient on coordinate (heterostructure includes into itself several layers) and temperature. The function $P(x, y, z, T)$ de- 
scribes dependences of limit of solubility of dopant on coordinate (heterostructure includes into itself several layers) and temperature. Parameter $\gamma \in[1,3]$ will be changed with changing of materials of heterostructure [10]. The function $V(x, y, z, t)$ describes distribution of concentration of radiation vacancies in space and time with the equilibrium distribution $V^{*}$. Dependence of dopant diffusion coefficient on concentration of the dopant has been described in details in Ref. [10]. Doping of materials by diffusion did not leads to generation radiation defects. In this situation $\zeta_{1}=\zeta_{2}=0$. We determine the spatio-temporal distributions of concentrations of radiation defects by solving the following system of equations $[11,12]$

$$
\begin{aligned}
& \frac{\partial I(x, y, z, t)}{\partial t}=\frac{\partial}{\partial x}\left[D_{I}(x, y, z, T) \frac{\partial I(x, y, z, t)}{\partial x}\right]+\frac{\partial}{\partial y}\left[D_{I}(x, y, z, T) \frac{\partial I(x, y, z, t)}{\partial y}\right]-k_{I, I}(x, y, z, T) \times \\
& \quad \times I^{2}(x, y, z, t)+\frac{\partial}{\partial z}\left[D_{I}(x, y, z, T) \frac{\partial I(x, y, z, t)}{\partial z}\right]-k_{I, V}(x, y, z, T) I(x, y, z, t) V(x, y, z, t) \\
& \frac{\partial V(x, y, z, t)}{\partial t}=\frac{\partial}{\partial x}\left[D_{V}(x, y, z, T) \frac{\partial V(x, y, z, t)}{\partial x}\right]+\frac{\partial}{\partial y}\left[D_{V}(x, y, z, T) \frac{\partial V(x, y, z, t)}{\partial y}\right]-k_{V, V}(x, y, z, T) \times \\
& \quad \times V^{2}(x, y, z, t)+\frac{\partial}{\partial z}\left[D_{V}(x, y, z, T) \frac{\partial V(x, y, z, t)}{\partial z}\right]-k_{I, V}(x, y, z, T) I(x, y, z, t) V(x, y, z, t)
\end{aligned}
$$

with boundary and initial conditions

$$
\begin{gathered}
\left.\frac{\partial \rho(x, y, z, t)}{\partial x}\right|_{x=0}=0,\left.\frac{\partial \rho(x, y, z, t)}{\partial x}\right|_{x=L_{x}}=0,\left.\frac{\partial \rho(x, y, z, t)}{\partial y}\right|_{y=0}=0,\left.\frac{\partial \rho(x, y, z, t)}{\partial y}\right|_{y=L_{y}}=0, \\
\left.\frac{\partial \rho(x, y, z, t)}{\partial z}\right|_{z=0}=0,\left.\frac{\partial \rho(x, y, z, t)}{\partial z}\right|_{z=L_{z}}=0, \rho(x, y, z, 0)=f_{\rho}(x, y, z) .
\end{gathered}
$$

Here $\rho=I, V$. The function $I(x, y, z, t)$ describes distribution of concentration of radiation interstitials in space and time. The functions $D_{\rho}(x, y, z, T)$ describes dependences of diffusion coefficients of point radiation defects on coordinate and temperature. Terms $V^{2}(x, y, z, t)$ and $I^{2}(x, y, z, t)$ describe generation of simplest complexes of radiation defects (divacancies and diinterstitials). The function $k_{I, V}(x, y, z, T)$ describes dependences of parameter of recombination on coordinate and temperature. The functions $k_{I, I}(x, y, z, T)$ and $k_{V, V}(x, y, z, T)$ describ dependences of the parameters of generation of divacancies and diinterstitials on coordinate and temperature.

We calculate distributions of concentrations of divacancies $\Phi_{V}(x, y, z, t)$ and dinterstitials $\Phi_{I}(x, y, z$, $t)$ in space and time as solution of the following system of equations $[11,12]$

$$
\begin{aligned}
& \frac{\partial \Phi_{I}(x, y, z, t)}{\partial t}=\frac{\partial}{\partial x}\left[D_{\Phi I}(x, y, z, T) \frac{\partial \Phi_{I}(x, y, z, t)}{\partial x}\right]+\frac{\partial}{\partial y}\left[D_{\Phi I}(x, y, z, T) \frac{\partial \Phi_{I}(x, y, z, t)}{\partial y}\right]+ \\
& +\frac{\partial}{\partial z}\left[D_{\Phi I}(x, y, z, T) \frac{\partial \Phi_{I}(x, y, z, t)}{\partial z}\right]+k_{I, I}(x, y, z, T) I^{2}(x, y, z, t)-k_{I}(x, y, z, T) I(x, y, z, t) \\
& \frac{\partial \Phi_{V}(x, y, z, t)}{\partial t}=\frac{\partial}{\partial x}\left[D_{\Phi V}(x, y, z, T) \frac{\partial \Phi_{V}(x, y, z, t)}{\partial x}\right]+\frac{\partial}{\partial y}\left[D_{\Phi V}(x, y, z, T) \frac{\partial \Phi_{V}(x, y, z, t)}{\partial y}\right]+
\end{aligned}
$$


International Journal in Foundations of Computer Science \& Technology (IJFCST) Vol.6, No.4, July 2016

$$
+\frac{\partial}{\partial z}\left[D_{\Phi V}(x, y, z, T) \frac{\partial \Phi_{V}(x, y, z, t)}{\partial z}\right]+k_{V, V}(x, y, z, T) V^{2}(x, y, z, t)-k_{V}(x, y, z, T) V(x, y, z, t)
$$

with the following boundary and initial conditions

$$
\begin{gathered}
\left.\frac{\partial \Phi_{\rho}(x, y, z, t)}{\partial x}\right|_{x=0}=0,\left.\frac{\partial \Phi_{\rho}(x, y, z, t)}{\partial x}\right|_{x=L_{x}}=0,\left.\frac{\partial \Phi_{\rho}(x, y, z, t)}{\partial y}\right|_{y=0}=0,\left.\frac{\partial \Phi_{\rho}(x, y, z, t)}{\partial y}\right|_{y=L_{y}}=0, \\
\left.\frac{\partial \Phi_{\rho}(x, y, z, t)}{\partial z}\right|_{z=0}=0,\left.\frac{\partial \Phi_{\rho}(x, y, z, t)}{\partial z}\right|_{z=L_{z}}=0, \Phi_{I}(x, y, z, 0)=f_{\Phi I}(x, y, z), \Phi_{V}(x, y, z, 0)=f_{\Phi V}(x, y, z) .(7)
\end{gathered}
$$

The functions $D_{\Phi \rho}(x, y, z, T)$ describe dependences of diffusion coefficients of the above complexes on coordinate and temperature. The functions $k_{I}(x, y, z, T)$ and $k_{V}(x, y, z, T)$ describe dependences of parameters of decay of these complexes on coordinate and temperature.

We calculate distributions of concentrations of dopant and radiation defects in space and time by using method of averaging of function corrections [13]. We consider the method with decreased quantity of iteration steps [14]. To decrease quantity of iteration steps we used solutions of linear Eqs. (1), (4) and (6) with averaged values of diffusion coefficients $D_{0 L}, D_{0 I}, D_{0 V}, D_{0 \Phi I}, D_{0 \Phi V}$ as initial-order approximations of the required concentrations. The solutions could be written as

$$
\begin{gathered}
C_{1}(x, y, z, t)=\frac{F_{0 C}}{L_{x} L_{y} L_{z}}+\frac{2}{L_{x} L_{y} L_{z}} \sum_{n=1}^{\infty} F_{n C} c_{n}(x) c_{n}(y) c_{n}(z) e_{n C}(t), \\
I_{1}(x, y, z, t)=\frac{F_{0 I}}{L_{x} L_{y} L_{z}}+\frac{2}{L_{x} L_{y} L_{z}} \sum_{n=1}^{\infty} F_{n I} c_{n}(x) c_{n}(y) c_{n}(z) e_{n I}(t), \\
V_{1}(x, y, z, t)=\frac{F_{0 C}}{L_{x} L_{y} L_{z}}+\frac{2}{L_{x} L_{y} L_{z}} \sum_{n=1}^{\infty} F_{n C} c_{n}(x) c_{n}(y) c_{n}(z) e_{n V}(t), \\
\Phi_{I 1}(x, y, z, t)=\frac{F_{0 \Phi_{I}}}{L_{x} L_{y} L_{z}}+\frac{2}{L_{x} L_{y} L_{z}} \sum_{n=1}^{\infty} F_{n \Phi_{I}} c_{n}(x) c_{n}(y) c_{n}(z) e_{n \Phi_{I}}(t), \\
\Phi_{V 1}(x, y, z, t)=\frac{F_{0 \Phi_{V}}}{L_{x} L_{y} L_{z}}+\frac{2}{L_{x} L_{y} L_{z}} \sum_{n=1}^{\infty} F_{n \Phi_{V}} c_{n}(x) c_{n}(y) c_{n}(z) e_{n \Phi_{V}}(t) .
\end{gathered}
$$

Here $e_{n \rho}(t)=\exp \left[-\pi^{2} n^{2} D_{0 \rho} t\left(\frac{1}{L_{x}^{2}}+\frac{1}{L_{y}^{2}}+\frac{1}{L_{z}^{2}}\right)\right], F_{n \rho}=\int_{0}^{L_{x}} c_{n}(u) \int_{0}^{L_{y}} c_{n}(v) \int_{0}^{L_{z}} c_{n}(v) f_{\rho}(u, v, w) d w d v d u, c_{n}(\chi)=\cos (\pi$ $\left.n \chi \mid L_{\chi}\right)$.

The approximations of concentrations of dopant and radiation defects with the second and higher orders have been calculated framework standard iterative procedure $[13,14]$. To use the procedure for calculation the $n$-order approximations one shall replace the functions $C(x, y, z, t), I(x, y, z, t)$, $V(x, y, z, t), \Phi_{I}(x, y, z, t), \Phi_{V}(x, y, z, t)$ in the right sides of the Eqs. (1), (4) and (6) on the following sums $\alpha_{n}+\rho_{n-1}(x, y, z, t)$. The replacement leads to the following result

$$
\begin{aligned}
& \frac{\partial C_{2}(x, y, z, t)}{\partial t}=\frac{\partial}{\partial x}\left(\left[1+\varsigma_{1} \frac{V(x, y, z, t)}{V^{*}}+\varsigma_{2} \frac{V^{2}(x, y, z, t)}{\left(V^{*}\right)^{2}}\right]\left\{1+\xi \frac{\left[\alpha_{2 C}+C_{1}(x, y, z, t)\right]^{\gamma}}{P^{\gamma}(x, y, z, T)}\right\} \times\right. \\
& \left.\times D_{L}(x, y, z, T) \frac{\partial C_{1}(x, y, z, t)}{\partial x}\right)+\frac{\partial}{\partial y}\left(D_{L}(x, y, z, T)\left[1+\varsigma_{1} \frac{V(x, y, z, t)}{V^{*}}+\varsigma_{2} \frac{V^{2}(x, y, z, t)}{\left(V^{*}\right)^{2}}\right] \times\right.
\end{aligned}
$$


International Journal in Foundations of Computer Science \& Technology (IJFCST) Vol.6, No.4, July 2016

$$
\begin{aligned}
& \left.\times\left\{1+\xi \frac{\left[\alpha_{2 C}+C_{1}(x, y, z, t)\right]^{\gamma}}{P^{\gamma}(x, y, z, T)}\right\} \frac{\partial C_{1}(x, y, z, t)}{\partial y}\right)+\frac{\partial}{\partial z}\left(D_{L}(x, y, z, T) \frac{\partial C_{1}(x, y, z, t)}{\partial z} \times\right. \\
& \left.\times\left[1+\varsigma_{1} \frac{V(x, y, z, t)}{V^{*}}+\varsigma_{2} \frac{V^{2}(x, y, z, t)}{\left(V^{*}\right)^{2}}\right]\left\{1+\xi \frac{\left[\alpha_{2 C}+C_{1}(x, y, z, t)\right]^{\gamma}}{P^{\gamma}(x, y, z, T)}\right\}\right) \\
& \frac{\partial I_{2}(x, y, z, t)}{\partial t}=\frac{\partial}{\partial x}\left[D_{I}(x, y, z, T) \frac{\partial I_{1}(x, y, z, t)}{\partial x}\right]+\frac{\partial}{\partial y}\left[D_{I}(x, y, z, T) \frac{\partial I_{1}(x, y, z, t)}{\partial y}\right]+ \\
& +\frac{\partial}{\partial z}\left[D_{I}(x, y, z, T) \frac{\partial I_{1}(x, y, z, t)}{\partial z}\right]-k_{I, V}(x, y, z, T)\left[\alpha_{2 I}+I_{1}(x, y, z, t)\right]\left[\alpha_{2 V}+V_{1}(x, y, z, t)\right]- \\
& -k_{I, I}(x, y, z, T)\left[\alpha_{2 I}+I_{1}(x, y, z, t)\right]^{2} \\
& \frac{\partial V_{2}(x, y, z, t)}{\partial t}=\frac{\partial}{\partial x}\left[D_{V}(x, y, z, T) \frac{\partial V_{1}(x, y, z, t)}{\partial x}\right]+\frac{\partial}{\partial y}\left[D_{V}(x, y, z, T) \frac{\partial V_{1}(x, y, z, t)}{\partial y}\right]+ \\
& +\frac{\partial}{\partial z}\left[D_{V}(x, y, z, T) \frac{\partial V_{1}(x, y, z, t)}{\partial z}\right]-k_{I, V}(x, y, z, T)\left[\alpha_{2 I}+I_{1}(x, y, z, t)\right]\left[\alpha_{2 V}+V_{1}(x, y, z, t)\right]- \\
& -k_{V, V}(x, y, z, T)\left[\alpha_{2 V}+V_{1}(x, y, z, t)\right]^{2} \\
& \frac{\partial \Phi_{I 2}(x, y, z, t)}{\partial t}=\frac{\partial}{\partial x}\left[D_{\Phi_{I}}(x, y, z, T) \frac{\partial \Phi_{I 1}(x, y, z, t)}{\partial x}\right]+\frac{\partial}{\partial y}\left[D_{\Phi_{I}}(x, y, z, T) \frac{\partial \Phi_{I 1}(x, y, z, t)}{\partial y}\right]+ \\
& +\frac{\partial}{\partial z}\left[D_{\Phi_{I}}(x, y, z, T) \frac{\partial \Phi_{I 1}(x, y, z, t)}{\partial z}\right]+k_{I, I}(x, y, z, T) I^{2}(x, y, z, t)-k_{I}(x, y, z, T) I(x, y, z, t) \\
& \frac{\partial \Phi_{V 2}(x, y, z, t)}{\partial t}=\frac{\partial}{\partial x}\left[D_{\Phi_{V}}(x, y, z, T) \frac{\partial \Phi_{V 1}(x, y, z, t)}{\partial x}\right]+\frac{\partial}{\partial y}\left[D_{\Phi_{V}}(x, y, z, T) \frac{\partial \Phi_{V 1}(x, y, z, t)}{\partial y}\right]+ \\
& +\frac{\partial}{\partial z}\left[D_{\Phi_{V}}(x, y, z, T) \frac{\partial \Phi_{V 1}(x, y, z, t)}{\partial z}\right]+k_{V, V}(x, y, z, T) V^{2}(x, y, z, t)-k_{V}(x, y, z, T) V(x, y, z, t) .
\end{aligned}
$$

Now we integrate left and right sides of Eqs.(8)-(10). The integration leads to the following results

$$
\begin{gathered}
C_{2}(x, y, z, t)=\frac{\partial}{\partial x}\left(\int_{0}^{t}\left[1+\varsigma_{1} \frac{V(x, y, z, \tau)}{V^{*}}+\varsigma_{2} \frac{V^{2}(x, y, z, \tau)}{\left.V^{*}\right)^{2}}\right]\left\{1+\xi \frac{\left[\alpha_{2 C}+C_{1}(x, y, z, \tau)\right]^{\gamma}}{P^{\gamma}(x, y, z, T)}\right\} \times\right. \\
\left.\times D_{L}(x, y, z, T) \frac{\partial C_{1}(x, y, z, \tau)}{\partial x}\right) d \tau+\frac{\partial}{\partial y}\left(\int_{0}^{t} D_{L}(x, y, z, T)\left[1+\varsigma_{1} \frac{V(x, y, z, \tau)}{V^{*}}+\varsigma_{2} \frac{V^{2}(x, y, z, \tau)}{\left(V^{*}\right)^{2}}\right] \times\right. \\
\left.\times\left\{1+\xi \frac{\left[\alpha_{2 C}+C_{1}(x, y, z, \tau)\right]^{\gamma}}{P^{\gamma}(x, y, z, T)}\right\} \frac{\partial C_{1}(x, y, z, \tau)}{\partial y} d \tau\right)+\frac{\partial}{\partial z}\left(\int_{0}^{t} D_{L}(x, y, z, T) \frac{\partial C_{1}(x, y, z, \tau)}{\partial z} \times\right. \\
\left.\times\left[1+\varsigma_{1} \frac{V(x, y, z, \tau)}{V^{*}}+\varsigma_{2} \frac{V^{2}(x, y, z, \tau)}{\left(V^{*}\right)^{2}}\right]\left\{1+\xi \frac{\left[\alpha_{2 C}+C_{1}(x, y, z, \tau)\right]^{\gamma}}{P^{\gamma}(x, y, z, T)}\right\} d \tau\right)+f_{C}(x, y, z)
\end{gathered}
$$


International Journal in Foundations of Computer Science \& Technology (IJFCST) Vol.6, No.4, July 2016

$$
\begin{aligned}
& I_{2}(x, y, z, t)=\frac{\partial}{\partial x}\left[\int_{0}^{t} D_{I}(x, y, z, T) \frac{\partial I_{1}(x, y, z, \tau)}{\partial x} d \tau\right]+\frac{\partial}{\partial y}\left[\int_{0}^{t} D_{I}(x, y, z, T) \frac{\partial I_{1}(x, y, z, \tau)}{\partial y} d \tau\right]+ \\
& +\frac{\partial}{\partial z}\left[\int_{0}^{t} D_{I}(x, y, z, T) \frac{\partial I_{1}(x, y, z, \tau)}{\partial z} d \tau\right]-\int_{0}^{t} k_{I, I}(x, y, z, T)\left[\alpha_{2 I}+I_{1}(x, y, z, \tau)\right]^{2} d \tau+f_{I}(x, y, z)- \\
& -\int_{0}^{t} k_{I, V}(x, y, z, T)\left[\alpha_{2 I}+I_{1}(x, y, z, \tau)\right]\left[\alpha_{2 V}+V_{1}(x, y, z, \tau)\right] d \tau \\
& V_{2}(x, y, z, t)=\frac{\partial}{\partial x}\left[\int_{0}^{t} D_{V}(x, y, z, T) \frac{\partial V_{1}(x, y, z, \tau)}{\partial x} d \tau\right]+\frac{\partial}{\partial y}\left[\int_{0}^{t} D_{V}(x, y, z, T) \frac{\partial V_{1}(x, y, z, \tau)}{\partial y} d \tau\right]+ \\
& \left.\times \frac{\partial V_{1}(x, y, z, \tau)}{\partial y} d \tau\right]+\frac{\partial}{\partial z}\left[\int_{0}^{t} D_{V}(x, y, z, T) \frac{\partial V_{1}(x, y, z, \tau)}{\partial z} d \tau\right]-\int_{0}^{t} k_{V, V}(x, y, z, T)\left[\alpha_{2 I}+V_{1}(x, y, z, \tau)\right]^{2} d \tau+ \\
& +f_{V}(x, y, z)-\int_{0}^{t} k_{I, V}(x, y, z, T)\left[\alpha_{2 I}+I_{1}(x, y, z, \tau)\right]\left[\alpha_{2 V}+V_{1}(x, y, z, \tau)\right] d \tau \\
& \Phi_{I 2}(x, y, z, t)=\frac{\partial}{\partial x}\left[\int_{0}^{t} D_{\Phi_{I}}(x, y, z, T) \frac{\partial \Phi_{I 1}(x, y, z, \tau)}{\partial x} d \tau\right]+\frac{\partial}{\partial y}\left[\int_{0}^{t} D_{\Phi_{I}}(x, y, z, T) \frac{\partial \Phi_{I 1}(x, y, z, \tau)}{\partial y} d \tau\right]+ \\
& +\frac{\partial}{\partial z}\left[\int_{0}^{t} D_{\Phi_{I}}(x, y, z, T) \frac{\partial \Phi_{I 1}(x, y, z, \tau)}{\partial z} d \tau\right]+\int_{0}^{t} k_{I, I}(x, y, z, T) I^{2}(x, y, z, \tau) d \tau+f_{\Phi_{I}}(x, y, z)- \\
& -\int_{0}^{t} k_{V}(x, y, z, T) V(x, y, z, \tau) d \tau \\
& \Phi_{V 2}(x, y, z, t)=\frac{\partial}{\partial x}\left[\int_{0}^{t} D_{\Phi_{V}}(x, y, z, T) \frac{\partial \Phi_{V 1}(x, y, z, \tau)}{\partial x} d \tau\right]+\frac{\partial}{\partial y}\left[\int_{0}^{t} D_{\Phi_{V}}(x, y, z, T) \frac{\partial \Phi_{V 1}(x, y, z, \tau)}{\partial y} d \tau\right]+ \\
& +\frac{\partial}{\partial z}\left[\int_{0}^{t} D_{\Phi_{V}}(x, y, z, T) \frac{\partial \Phi_{V 1}(x, y, z, \tau)}{\partial z} d \tau\right]+\int_{0}^{t} k_{V, V}(x, y, z, T) V^{2}(x, y, z, \tau) d \tau+f_{\Phi_{V}}(x, y, z)- \\
& -\int_{0}^{t} k_{V}(x, y, z, T) V(x, y, z, \tau) d \tau \text {. }
\end{aligned}
$$

We calculate average values of the second-orders approximations of the above concentrations by using the following relations $[13,14]$

$$
\alpha_{2 \rho}=\frac{1}{\Theta L_{x} L_{y} L_{z}} \int_{0}^{\Theta} \int_{0}^{L_{x}} \int_{0}^{L_{y}} \int_{0}^{L_{z}}\left[\rho_{2}(x, y, z, t)-\rho_{1}(x, y, z, t)\right] d z d y d x d t
$$

Relations for the required average values $\alpha_{2 \rho}$ have been calculated through substitution of relations $(8 a)-(10 a)$ into relation (11) and could be written as

$$
\begin{gathered}
\alpha_{2 C}=\frac{1}{L_{x} L_{y} L_{z}} \int_{0}^{L_{x}} \int_{0}^{L_{y}} \int_{0}^{L_{z}} f_{C}(x, y, z) d z d y d x \\
\alpha_{2 I}=\frac{1}{2 A_{I I 00}}\left\{\left(1+A_{I V 01}+A_{I I 10}+\alpha_{2 V} A_{I V 00}\right)^{2}-4 A_{I I 00}\left[A_{I V 11}-\frac{1}{L_{x} L_{y} L_{z}} \int_{0}^{L_{x}} \int_{0}^{L_{y}} \int_{0}^{L_{z}} f_{I}(x, y, z) d z d y d x+\right.\right.
\end{gathered}
$$


International Journal in Foundations of Computer Science \& Technology (IJFCST) Vol.6, No.4, July 2016

$$
\begin{gathered}
\left.\left.+\alpha_{2 V} A_{I V 10}-A_{I I 20}\right]\right]^{\frac{1}{2}}-\frac{1+A_{I V 01}+A_{I I 10}+\alpha_{2 V} A_{I V 00}}{2 A_{I I 00}} \\
\alpha_{2 V}=\frac{1}{2 B_{4}} \sqrt{\frac{\left(B_{3}+A\right)^{2}}{4}-4 B_{4}\left(y+\frac{B_{3} y-B_{1}}{A}\right)}-\frac{B_{3}+A}{4 B_{4}} .
\end{gathered}
$$

Here $A_{a b i j}=\frac{1}{\Theta L_{x} L_{y} L_{z}} \int_{0}^{\Theta}(\Theta-t) \iint_{0}^{L_{L}} \int_{0}^{L_{y}} \int_{0}^{L_{z}} k_{a, b}(x, y, z, T) I_{1}^{i}(x, y, z, t) V_{1}^{j}(x, y, z, t) d z d y d x d t, B_{4}=A_{I V 00}^{2} A_{I V 00}^{2}-$ $-2\left(A_{I V 00}^{2}-A_{I I 00} A_{V V 00}\right)^{2}, B_{3}=A_{I V 00} A_{I V 00}^{2}+A_{I V 00} A_{I I 0} A_{I V 00}^{2}-4 A_{I V 10} A_{I I 00} A_{I V 00}^{2}+2 A_{I V 00} A_{I V 01} A_{I V 00}^{2}+A_{I V 01} \times$ $\times A_{I V 00}^{3}-4\left(A_{I V 00}^{2}-A_{I I 00} A_{V V 00}\right)\left[2 A_{I V 01} A_{I V 00}+2 A_{I V 00}\left(1+A_{I V 01}+A_{I I 0}\right)-2 A_{I I 00}\left(A_{I V 10}+A_{V V 10}+1\right)\right], B_{2}=$ $=A_{I V 00}^{2}\left\{\left(1+A_{I V 01}+A_{I I 10}\right)^{2}+A_{I V 00}^{2} A_{I V 01}^{2}+2 A_{I V 00} A_{I V 00}\left(A_{I V 00}+A_{I V 00} A_{I V 01}+A_{I V 00} A_{I I 10}-4 A_{I V 10} A_{I I 00}\right)-\right.$ $\left.-4 A_{I I 00}\left[A_{I V 11}-A_{I I 20}-\frac{1}{L_{x} L_{y} L_{z}} \int_{0}^{L_{x}} \int_{0}^{L_{2} L_{z}} \int_{0} f_{I}(x, y, z) d z d y d x\right]\right\}\left\{\left[2 A_{I V 01} A_{I V 00}+2 A_{I V 00}\left(1+A_{I V 01}+A_{I I 10}\right)-\right.\right.$ $\left.-2 A_{I I 00}\left(A_{I V 10}+A_{V V 10}+1\right)\right]^{2}+2\left[A_{I V 01}\left(1+A_{I V 01}+A_{I I 10}\right)+\frac{2}{L_{x} L_{y} L_{z}} \int_{0}^{L_{x}} \int_{0}^{L_{y} L_{2}} \int_{0} f_{V}(x, y, z) d z d y d x-2 A_{I I 00}\left(A_{V V 20}-\right.\right.$ $\left.\left.\left.-A_{I V 11}\right)+A_{I V 01}\left(1+A_{I V 01}+A_{I I 10}\right)\right]\left[2 A_{I V 00}\left(1+A_{I V 01}+A_{I I 0}\right)+2 A_{I V 01} A_{I V 00}-2 A_{I I 00}\left(A_{I V 10}+A_{V V 10}+1\right)\right]\right\}$, $B_{1}=2 A_{I V 00} A_{I V 01}\left(1+A_{I V 01}+A_{I I 10}\right)^{2}-8\left[A_{I V 11}-A_{I I 20}-\frac{1}{L_{x} L_{y} L_{z}} \int_{0}^{L_{L}} \int_{0}^{L_{\nu} L_{z}} \int_{0} f_{I}(x, y, z) d z d y d x\right]+A_{I V 00} A_{I V 01} \times$ $\times A_{I I 00}+A_{I V 01}^{2}\left(A_{I V 00}+A_{I V 00} A_{I V 01}+A_{I V 00} A_{I I 10}-4 A_{I V 10} A_{I I 00}\right)-2\left[\frac{2 A_{I I 00}}{L_{x} L_{y} L_{z}} \int_{0}^{L_{x} L_{y} L_{z}} \int_{0} f_{I}(x, y, z) d z d y d x+\right.$ $\left.+A_{I V 01}\left(1+A_{I V 01}+A_{I I 10}\right)-2 A_{I I 00}\left(A_{V V 20}-A_{I V 11}\right)+A_{I V 01}\left(1+A_{I V 01}+A_{I I 0}\right)\right]\left[2 A_{I V 00}\left(1+A_{I V 01}+A_{I I 10}\right)-\right.$ $\left.-2\left(A_{I V 10}+A_{V V 10}+1\right) A_{I I 00}+2 A_{I V 01} A_{I V 00}\right], B_{0}=\left[A_{I I 20}+\frac{1}{L_{x} L_{y} L_{z}} \int_{0}^{L_{x}} \int_{0}^{L_{y} L_{z}} \int_{0} f_{I}(x, y, z) d z d y d x-A_{I V 11}\right] \times$ $\times 4 A_{I I 00} A_{I V 01}^{2}+A_{I V 01}^{2}\left(1+A_{I V 01}+A_{I I 0}\right)^{2}-\left[\frac{2 A_{I I 00}}{L_{x} L_{y} L_{z}} \int_{0}^{L_{x}} \int_{0}^{L_{y}} \int_{0}^{L_{z}} f_{V}(x, y, z) d z d y d x+A_{I V 01}\left(1+A_{I V 01}+A_{I I 10}\right)-\right.$ $\left.-2 A_{I I 00}\left(A_{V V 20}-A_{I V 11}\right)+A_{I V 01}\left(1+A_{I V 01}+A_{I I 10}\right)\right]^{2}, y=\sqrt[3]{\sqrt{q^{2}+p^{3}}-q}-\sqrt[3]{\sqrt{q^{2}+p^{3}}+q}+\frac{B_{2}}{6}, q=\frac{B_{2}}{48} \times$ $\times\left(2 B_{1} B_{3}-8 B_{0}\right)+\frac{B_{2}^{3}}{216}+\frac{B_{0}\left(4 B_{2}-B_{3}^{2}\right)-B_{1}^{2}}{8}, p=\frac{3\left(2 B_{1} B_{3}-8 B_{0}\right)-2 B_{2}^{2}}{72}, A=\sqrt{8 y+B_{3}^{2}-4 B_{2}}$, $\alpha_{2 \Phi_{I}}=A_{I I 20}-\frac{1}{\Theta L_{x} L_{y} L_{z}} \int_{0}^{\Theta}(\Theta-t) \int_{0}^{L_{x} L_{y}} \int_{0}^{L_{z}} \int_{0}^{k_{I}} k_{I}(x, y, z, T) I(x, y, z, t) d z d y d x d t+$ $+\frac{1}{L_{x} L_{y} L_{z}} \int_{0}^{L_{x} L_{y}} \int_{0}^{L_{z}} \int_{0}^{L_{\Phi I}} f_{\Phi}(x, y, z) d z d y d x$

$\alpha_{2 \Phi_{V}}=A_{V V 20}-\frac{1}{\Theta L_{x} L_{y} L_{z}} \int_{0}^{\Theta}(\Theta-t) \int_{0}^{L_{x} L_{y}} \int_{0}^{L_{z}} \int_{0} k_{V}(x, y, z, T) V(x, y, z, t) d z d y d x d t+$ $+\frac{1}{L_{x} L_{y} L_{z}} \int_{0}^{L_{x}} \int_{0}^{L_{y}} \int_{0}^{L_{z}} f_{\Phi V}(x, y, z) d z d y d x$. 
After the substitution one can obtain equation for parameter $\alpha_{2 C}$. Solution of the equation will be different for different values of parameter $\gamma$. We analyzed distributions of concentrations of dopant and radiation defects in space and time by using the second-order approximations framework the method of averaging of function corrections with decreased quantity of iterative steps. The approximations are usually enough good approximation to make qualitative analysis and obtain some quantitative results. We check our analytical results by comparison with results of numerical simulation.

\section{DISCUSSION}

In this section we analyzed spatio-temporal distribution of concentration of infused (see Fig. $2 a$ ) and implanted (see Fig. $2 b$ ) dopants in the considered epitaxial layer. Annealing time is the same for the each curve framework each figure. Increasing of difference between values of dopant diffusion coefficient in layers of heterostructure corresponds to increasing of number of curves on these figures. One can find from these figures, that inhomogeneity of heterostructure leads to increasing of gradient of concentration of dopant at absolute value in direction, which is perpendicular to the interface. In this situation one can find decreasing of dimensions of transistors framework the considered element SRAM. One can also find increasing homogeneity of distribution of concentrations of dopants in doped areas.

With increasing annealing time gradient of concentration of dopant near interface between epitaxial layer and substrate decreases at absolute value. Decreasing of value of annealing time leads to increasing of inhomogeneity of distribution of concentration of dopant (see Figs. $3 a$ for diffusion type of doping and $3 b$ for ion type of doping). We determine the compromise value of annealing time framework recently introduced criterion [15-22]. To use the criterion we approximate distribution of concentration of dopant by idealized step-wise function $\psi(x, y, z)$. Compromise value of annealing time we calculate by minimization of the mean-squared error

$$
U=\frac{1}{L_{x} L_{y} L_{z}} \int_{0}^{L_{L}} \int_{0}^{L_{y} L_{z}} \int_{0}[C(x, y, z, \Theta)-\psi(x, y, z)] d z d y d x
$$

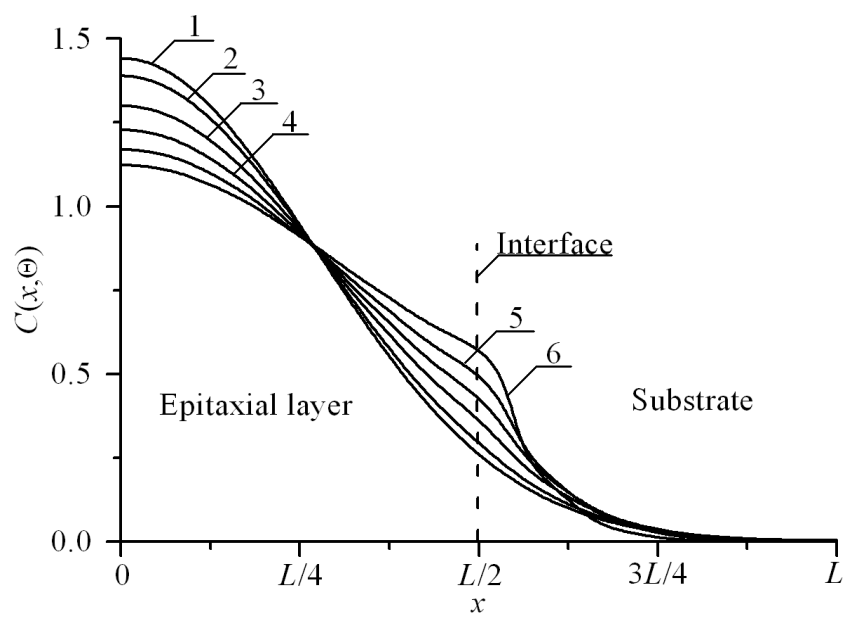

Fig.2a. Distributions of concentration of dopant, which has been infused in the considered heterostructure under condition, when value of dopant diffusion coefficient in epitaxial layer is larger, than value of dopant diffusion coefficient in substrate. Number of curves increases with increasing of difference between values of dopant diffusion coefficient 
International Journal in Foundations of Computer Science \& Technology (IJFCST) Vol.6, No.4, July 2016

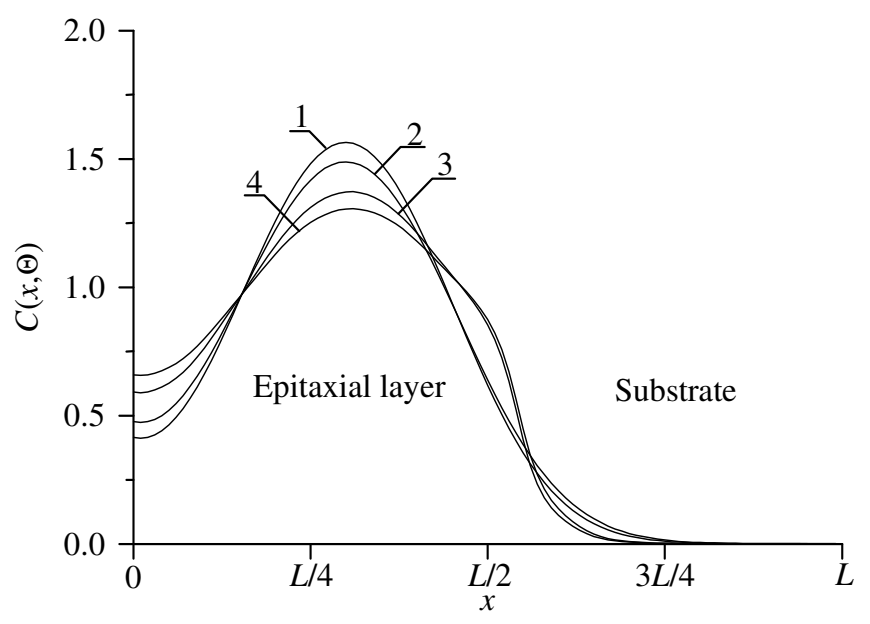

Fig.2b. Distributions of concentration of dopant, which has been implanted in the considered heterostructure under condition, when value of dopant diffusion coefficient in epitaxial layer is larger, than value of dopant diffusion coefficient in substrate. Curves 1 and 3 are distributions of concentration of dopant in homogenous sample at annealing time $\Theta=0.0048\left(L_{x}{ }^{2}+L_{y}{ }^{2}+L_{z}{ }^{2}\right) / D_{0}$. Curves 1 and 3 are distributions of concentration of dopant in heterostructure at annealing time $\Theta=0.0057\left(L_{x}{ }^{2}+L_{y}{ }^{2}+L_{z}{ }^{2}\right) / D_{0}$.

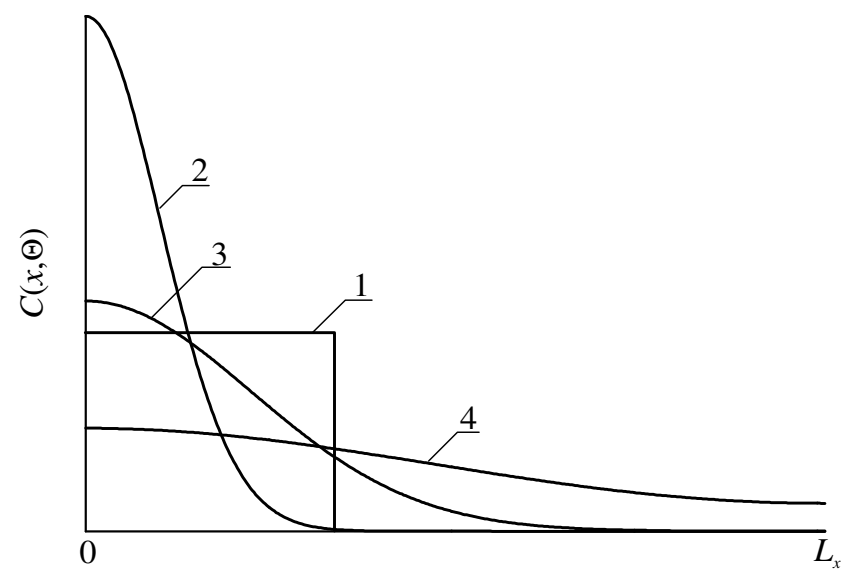

Fig. $3 a$. Distributions of concentration of infused dopant in heterostructure. Curve 1 is the idealized distribution of dopant. Curves 2-4 are the real distributions of concentration of dopant in heterostructure. Number of curves increases with increasing of annealing time 


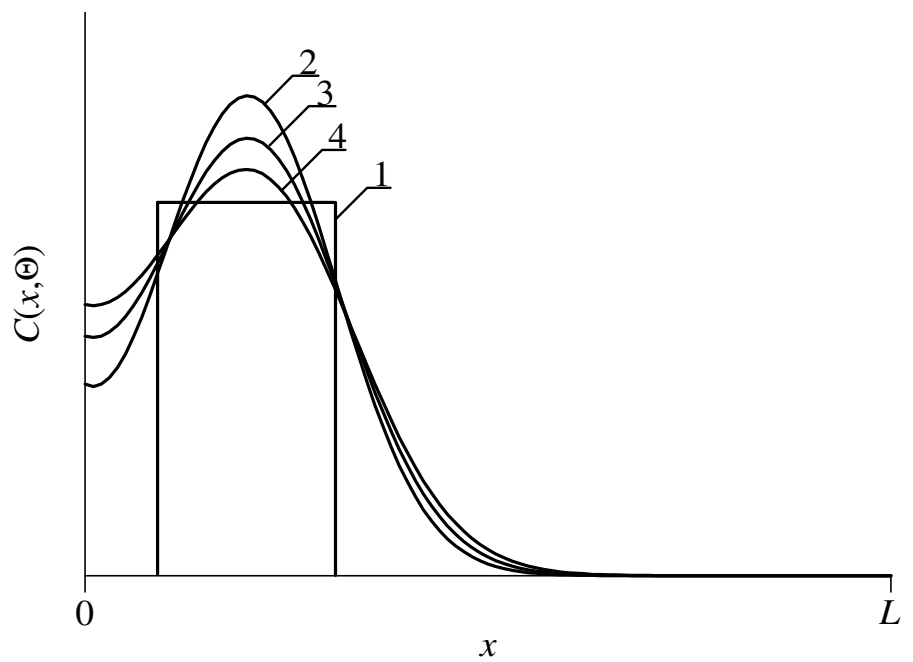

Fig. $3 b$. Distributions of concentration of implanted dopant in heterostructure. Curve 1 is the idealized distribution of dopant. Curves 2-4 are the real distributions of concentration of dopant in heterostructure.

Number of curves increases with increasing of annealing time

Optimal values of annealing time as functions of parameters are presented on Figs. 4. Fig. $4 a$ corresponds to diffusion type of doping. Fig. $4 b$ corresponds to ion type of doping. After ion implantation radiation defects should be annealed. Distribution of concentration of dopant will be spreads during the annealing. Distribution of dopant achieves appropriate interfaces between layers of heterostructures in the ideal case. If dopant have not enough time to achieve the interface, additional annealing of dopant attracted an interest, which will be smaller, than for infused dopant. However it should be noted, that ion type of doping leads to decreasing of mismatchinduced stress in heterostructure [23].

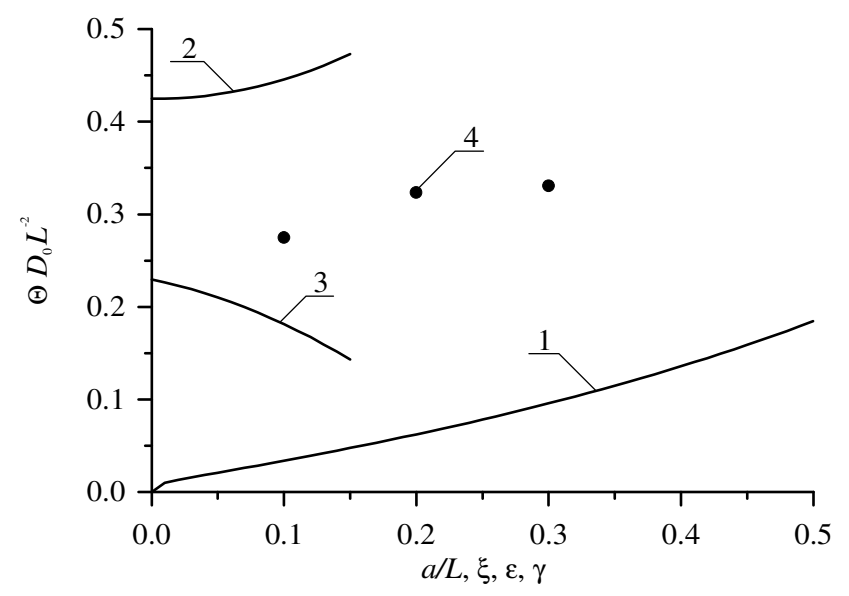

Fig.4a. Optimal annealing time of infused dopant as a function of different parameters. Curve 1 describes dependence of optimal annealing time on $a / L$ at $\xi=\gamma=0$ and equal to each other values of dopant diffusion coefficient in all parts of heterostructure. Curve 2 describes dependence of optimal annealing time on $\varepsilon$ at $a / L=1 / 2$ and $\xi=\gamma=0$. Curve 3 describes dependence of optimal annealing time on $\xi$ for $a / L=1 / 2$ and $\varepsilon=\gamma=0$.

Curve 4 describes dependence of optimal annealing time on $\gamma$ at $a / L=1 / 2$ and $\varepsilon=\xi=0$ 


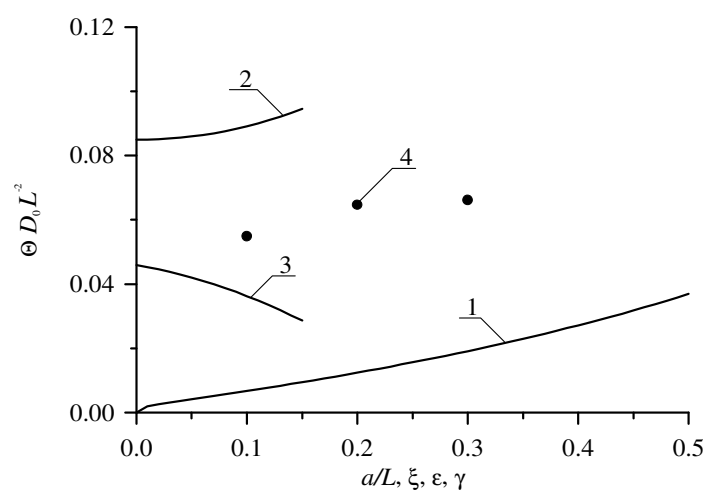

Fig.4b. Optimal annealing time of implanted dopant as a function of different parameters. Curve 1 describes dependence of optimal annealing time on $a / L$ at $\xi=\gamma=0$ and equal to each other values of dopant diffusion coefficient in all parts of heterostructure. Curve 2 describes dependence of optimal annealing time on $\varepsilon$ at $a / L=1 / 2$ and $\xi=\gamma=0$. Curve 3 describes dependence of optimal annealing time on $\xi$ for $a / L=1 / 2$ and $\varepsilon=\gamma=0$. Curve 4 describes dependence of optimal annealing time on $\gamma$ at $a / L=1 / 2$ and $\varepsilon=\xi=0$

\section{CONCLUSIONS}

In this paper we introduced an analytical approach to prognosis of concentrations of infused and implanted dopants. By using the approach we analyzed changing of these concentrations during manufacturing field-effect transistors framework the element SRAM. We formulate recommendations for optimization of annealing to decrease dimensions transistors and to increase their dimensions.

\section{ACKNOWLEDGEMENTS}

This work is supported by the agreement of August 27, 2013 № 02.B.49.21.0003 between The Ministry of education and science of the Russian Federation and Lobachevsky State University of Nizhni Novgorod, educational fellowship for scientific research of Government of Russian and educational fellowship for scientific research of Government of Nizhny Novgorod region of Russia.

\section{REFERENCES}

[1] K.K. Ong, K.L. Pey, P.S. Lee, A.T.S. Wee, X.C. Wang, Y.F. Chong. Dopant distribution in the recrystallization transient at the maximum melt depth induced by laser annealing. Appl. Phys. Lett. 89 (17), 172111-172114 (2006).

[2] H.T. Wang, L.S. Tan, E. F. Chor. Pulsed laser annealing of Be-implanted GaN. J. Appl. Phys. 98 (9), 094901-094905 (2006).

[3] Yu.V. Bykov, A.G. Yeremeev, N.A. Zharova, I.V. Plotnikov, K.I. Rybakov, M.N. Drozdov, Yu.N. Drozdov, V.D. Skupov. Diffusion processes in semiconductor structures during microwave annealing. Radiophysics and Quantum Electronics. Vol. 43 (3). P. 836-843 (2003).

[4] V.V. Kozlivsky. Modification of semiconductors by proton beams (Nauka, Sant-Peterburg, 2003, in Russian).

[5] V.L. Vinetskiy, G.A. Kholodar', Radiative physics of semiconductors. ("Naukova Dumka", Kiev, 1979, in Russian).

[6] G. Volovich. Modern chips UM3Ch class D manufactured by firm MPS. Modern Electronics. Issue 2. P. 10-17 (2006).

[7] A. Kerentsev, V. Lanin. Constructive-technological features of MOSFET-transistors. Power Electronics. Issue 1. P. 34 (2008). 
[8] A.O. Ageev, A.E. Belyaev, N.S. Boltovets, V.N. Ivanov, R.V. Konakova, Ya.Ya. Kudrik, P.M. Litvin, V.V. Milenin, A.V. Sachenko. Au-TiBx-n-6H-SiC Schottky barrier diodes: the features of current flow in rectifying and nonrectifying contacts. Semiconductors. Vol. 43 (7). P. 897-903 (2009).

[9] N.I. Volokobinskaya, I.N. Komarov, T.V. Matyukhina, V.I. Reshetnikov, A.A. Rush, I.V. Falina, A.S. Yastrebov. Investigation of technological processes of manufacturing of the bipolar power highvoltage transistors with a grid of inclusions in the collector region. Semiconductors. Vol. 35 (8). P. 1013-1017 (2001).

[10] Ch. Tanaka, M. Saitoh, K. Ota. T. Numata. Analysis of static noise margin improvement for low voltage SRAM composed of nano-scale MOSFETs with ideal subthreshold factor and small variability. Solid-State Electronics. Vol. 109 (1). P. 58-62 (2015).

[11] Z.Yu. Gotra. Technology of microelectronic devices (Radio and communication, Moscow, 1991).

[12] P.M. Fahey, P.B. Griffin, J.D. Plummer. Point defects and dopant diffusion in silicon. Rev. Mod. Phys. V. 61 (2). P. 289-388 (1989).

[13] Yu.D. Sokolov. About the definition of dynamic forces in the mine lifting. Applied Mechanics. Vol. 1 (1). P. 23-35 (1955).

[14] E.L. Pankratov. Dynamics of delta-dopant redistribution during heterostructure growth. The European Physical Journal B. V. 57 (3). P. 251-256 (2007).

[15] E.L. Pankratov. Dopant diffusion dynamics and optimal diffusion time as influenced by diffusioncoefficient nonuniformity. Russian Microelectronics. V.36 (1). P. 33-39 (2007).

[16] E.L. Pankratov. Redistribution of dopant during annealing of radiative defects in a multilayer structure by laser scans for production an implanted-junction rectifiers.Int. J. Nanoscience. Vol. 7 (4-5). P. 187-197 (2008).

[17] E.L. Pankratov. Decreasing of depth of implanted-junction rectifier in semiconductor heterostructure by optimized laser annealing. J. Comp. Theor. Nanoscience. Vol. 7 (1). P. 289-295 (2010).

[18] E.L. Pankratov, E.A. Bulaeva. An approach to decrease dimensions of field-effect transistors without p-n-junctions. Int. J. Mod. Phys. B. Vol. 28 (27). P. 1450190-1-1450190-17 (2014).

[19] E.L. Pankratov, E.A. Bulaeva. An approach to manufacture of bipolar transistors in thin film structures. On the method of optimization. Int. J. Micro-Nano Scale Transp. Vol. 4 (1). P. 17-31 (2014).

[20] E.L. Pankratov, E.A. Bulaeva. Increasing of sharpness of diffusion-junction heterorectifier by using radiation processing. Int. J. Nanoscience. Vol. 11 (5). P. 1250028-1250035 (2012).

[21] E.L. Pankratov, E.A. Bulaeva. An approach to decrease dimentions of logical elements based on bipolar transistor. Int. J. Comp. Sci. Appl. Vol. 5 (4). P. 1-18 (2015).

[22] E.L. Pankratov, E.A. Bulaeva. Nano Science and Nano Technology: An Indian Journal. Vol. 9 (4). P. 43-60 (2015).

[23] E.L. Pankratov, E.A. Bulaeva. Decreasing of mechanical stress in a semiconductor heterostructure by radiation processing. J. Comp. Theor. Nanoscience. Vol. 11 (1). P. 91-101 (2014).

\section{AUTHORS}

Pankratov Evgeny Leonidovich was born at 1977. From 1985 to 1995 he was educated in a secondary school in Nizhny Novgorod. From 1995 to 2004 he was educated in Nizhny Novgorod State University: from 1995 to 1999 it was bachelor course in Radiophysics, from 1999 to 2001 it was master course in Radiophysics with specialization in Statistical Radiophysics, from 2001 to 2004 it was PhD course in Radiophysics. From 2004 to 2008 E.L. Pankratov was a leading technologist in Institute for Physics of Microstructures. From 2008 to 2012 E.L. Pankratov was a senior lecture/Associate Professor of Nizhny Novgorod State University of Architecture and Civil Engineering. 2012-2015 Full Doctor course in Radiophysical Department of Nizhny Novgorod State University. Since 2015 E.L. Pankratov is an Associate Professor of Nizhny Novgorod State University. He has 155 published papers in area of his researches.

Bulaeva Elena Alexeevna was born at 1991. From 1997 to 2007 she was educated in secondary school of village Kochunovo of Nizhny Novgorod region. From 2007 to 2009 she was educated in boarding school "Center for gifted children". From 2009 she is a student of Nizhny Novgorod State University of Architecture and Civil Engineering (spatiality "Assessment and management of real estate"). At the same time she is a student of courses "Translator in the field of professional communication" and "Design (interior art)" in the University. Since 2014 E.A. Bulaeva is in a PhD program in Radiophysical Department of Nizhny Novgorod State University. She has 110 published papers in area of her researches. 